\begin{tabular}{|c|c|c|}
\hline 150 150 & $\begin{array}{c}\text { Bulletin of Pharmaceutical Sciences } \\
\text { Assiut University }\end{array}$ & 0 \\
\hline 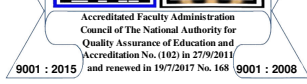 & $\begin{array}{c}\text { Website: http://lbpsa.journals.ekb.eg/ } \\
\text { e-mail: bullpharm@aun.edu.eg }\end{array}$ & $\begin{array}{c}\text { BULL. PHARM. SCI } \\
\text { Assiut Univ. }\end{array}$ \\
\hline
\end{tabular}

\title{
PRELIMINARY INVESTIGATION ON THE PRODUCTION OF SURFACE ACTIVE COMPOUNDS WITH ANTIMICROBIAL AND EMULSIFYING PROPERTIES BY BACILLUS CLAUSII AND LACTOBACILLUS RHAMNOSUS PROBIOTIC STRAINS
}

\author{
Tiago Santos Fonseca ${ }^{1}$, Renan Martins dos Santos ${ }^{1,2}$ and Marcus Vinícius Dias-Souza ${ }^{1,2 *}$ \\ ${ }^{1}$ Pitágoras College, Ipatinga, MG, Brazil \\ ${ }^{2}$ Integrated Pharmacology and Drug Interactions Research Group (GPqFAR), Brazil
}

\begin{abstract}
Surface active compounds (SACs) of bacterial origin are amphiphilic heteropolymers that may alter the interaction of gas-liquid, solid-liquid, and immiscible liquids surfaces, decrease the surface and interfacial tensions of liquids, and form stable emulsions. SACs are divided in two main groups: low molecular weight, the biosurfactants, and high molecular weight, the bioemulsifiers. Probiotics are live microorganisms that, when administered in determined levels, provide benefits to the host. They can produce SACs with several properties, such as the emulsification and solubilization of substrates and antimicrobial activity. Here we show that the cell-free supernatant of commercial probiotic strains of Bacillus clausii (formerly Bacillus subtilis), a spore-forming species that can tolerate biliary salts and survive at acid gastric conditions, and Lactobacillus rhamnosus, a lactic acid facultative heterofermentative bacterial species, are able to emulsify apolar fluids including gasoline fuel, suggesting the presence of biomolecules that could be explored for bioremediation. L. rhamnosus also presented antimicrobial activity, whereas Bacillus clausii did not.
\end{abstract}

\section{INTRODUCTION}

Probiotics are live bacterial or fungal microorganisms that, when administered in determined levels, provide varied benefits to the host ${ }^{1}$. Their effects on gut health and immune system are widely investigated, being their primary application as pharmaceutical formulations. Bacillus clausii and Lactobacillus rhamnosus strains are explored for commercial purposes in this context. $B$. clausii (formerly Bacillus subtilis) is a sporeforming species that can tolerate biliary salts and survive acid gastric conditions, and is commercially explored as a probiotic strain to manage diarrhea ${ }^{2}$. Lactobacillus rhamnosus is a lactic acid facultative heterofermentative species that can be isolated not only of dairy products, but also vaginal secretion, fermented beverages and human feces ${ }^{3}$. Phylogenetic studies clustered B. clausii with other probiotic
Bacillus strains, and clustered L. rhamnosus with other probiotic Lactobacillus species such as L. $\operatorname{casei}^{2 \& 3}$.

The mechanisms by which probiotics would provide benefits for the gut remain not totally clear. However, colonization of mucosal tissues (thus inhibiting pathogen attachment), immunomodulation, production of enzymes such as lactase, production of bacteriocins, which are antimicrobial peptides produced by bacteria, and production of biosurfactants, amphiphilic molecules that can reduce surface tension and present antimicrobial activity, have been used to explain some observations ${ }^{4}$. Biosurfactants are of special interest, as they could benefit other fields such as cosmetology and ecology, the last one especially for bioremediation purposes.

Here we show that two commercial probiotic strains - B.clausii and L. rhamnosus are able to produce surface active compounds

Received in 5/1/2021 \& Accepted in 11/2/2021 
with the ability to emulsify immiscible liquids and to reduce water surface tension, a topic poorly addressed by the currently available studies. Their antimicrobial potential was tested as well.

\section{MATERIALS AND METHODS}

\section{Strains and cultivation conditions}

B. clausii was purchased as a pharmaceutical formulation of spores prepared in water (Enterogermina, Sanofi-Aventis, France) and L. rhamnosus was purchased as lyophilized powder (Florien, Brazil). B. clausii was cultured in BHI broth (Difco, Becton Dickinson, USA), in aerobic conditions, at $37^{\circ} \mathrm{C}$. L. rhamnosus was cultured in MRS broth (Difco), replacing glucose for lactose, in anaerobic conditions, at $37^{\circ} \mathrm{C}$. After an overnight primary growth for activation of the strains, the bacteria were transferred to fresh medium as to reach a $0.5 \mathrm{McFarland}$ turbidity standard (optical density $=1$ at $600 \mathrm{~nm}$, checked by spectrophotometry), and kept for 24, 48 and $72 \mathrm{hrs}$, for $37^{\circ} \mathrm{C}$, without agitation, in glass flasks (B. clausii in aerobic conditions and $L$. rhamnosus in anaerobic conditions). After each time point, aliquots of each culture media were collected and centrifuged in home temperature (30 $\mathrm{min}, 3400 \mathrm{RPM}$ ) to obtain separated cell-free supernatant (CFS) for each species, which were tested separately for emulsifying and antimicrobial potentials.

\section{Emulsifying activity}

The emulsifying activity of each CFS was assessed using the $\mathrm{E}_{24}$ (emulsification index) method, as described by Monteiro et al. ${ }^{5}$, using aliquots of each CFS and hydrophobic substrates (Table 1 and Fig. 1) in a 2:3 proportion. The stability of the emulsions was monitored for two weeks after the experiments. We assessed differences on the natural tension of water with each CFS in a 9:1 proportion using the drop collapse method as previously described $^{6}$.

Table 1: Hydrophobic substrates for emulsification tests.

\begin{tabular}{|c|c|}
\hline Hydrophobic substrate & Details \\
\hline Hexane & Analytical grade (Hexis, Brazil) \\
\hline Gasoline (fuel) & Purchased locally \\
\hline Sunflower oil & Culinary grade, purchased locally \\
\hline Mineral oil & Pharmaceutical grade (Farmax, Brazil) \\
\hline
\end{tabular}

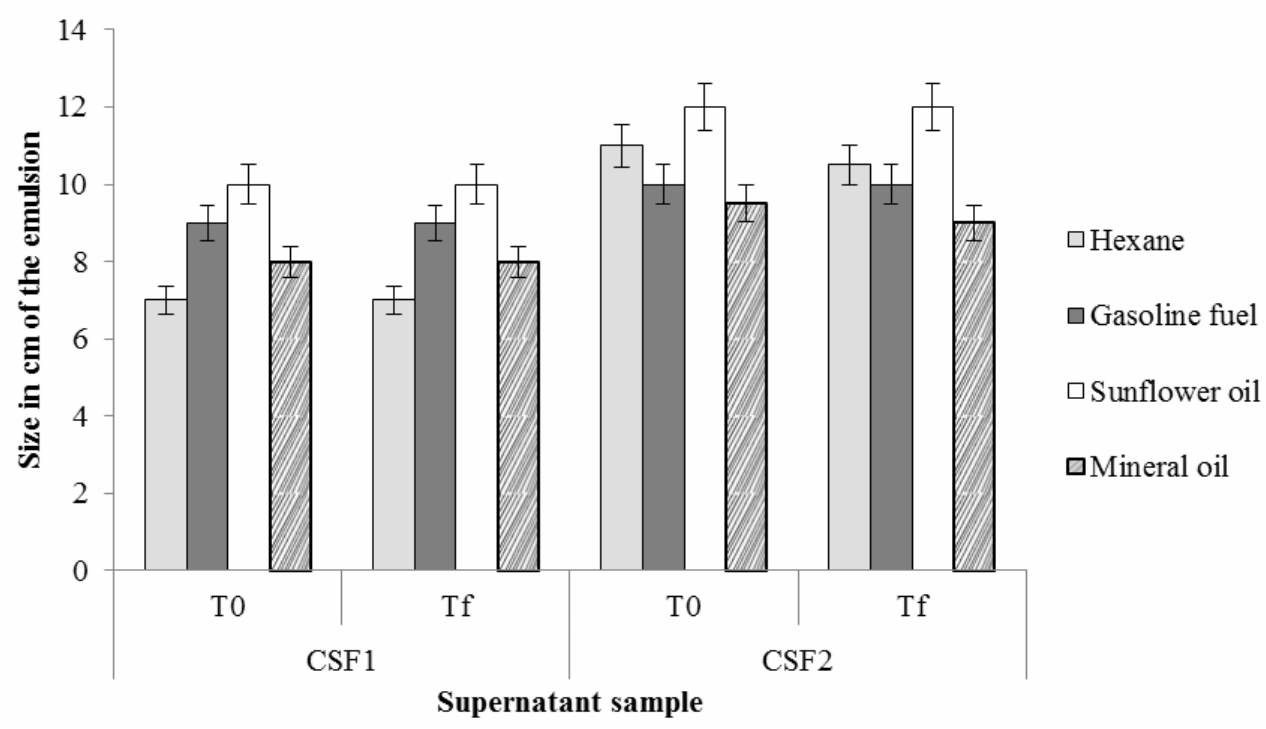

Fig.1: Results of the emulsification assay $\left(\mathrm{E}_{24}\right)$. T0: emulsion size right after the experiment. Tf: emulsion size right after 15 days. CFS1: supernatant obtained from B. clausii, CFS2: supernatant obtained from $L$. rhamnosus. 


\section{Antimicrobial activity tests}

We conducted semi-quantitative and qualitative assays with clinical isolates of Staphylococcus aureus (from catheter tips), Pseudomonas aeruginosa (from tracheal secretions) and Escherichia coli (uropathogenic strains), two strains of each species, all belonging to the microorganism collection of Pitágoras College ( $P$. aeruginosa: strains $\mathrm{n} \# 5$ and $\mathrm{n \# 6} ; E$. coli: strains $\mathrm{n \# 1}$ and

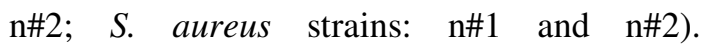
Overnight-grown bacterial isolates were cultured in nutrient broth, and 0.5 McFarland suspensions were prepared in fresh medium using $\mathrm{OD}_{600}$ spectrophotometric readings for the assays ${ }^{7}$.

Semi-quantitative assays were conducted in 96 wells polystyrene plates as follows: each CFS was serially diluted in sterile saline (up to $1 / 128$ dilution) in a total of $100 \mu \mathrm{L}$. A total of $100 \mu \mathrm{L}$ of each bacterial suspension with adjusted turbidity to 0.5 McFarland scale (using fresh sterile media) were than added. Plates were then incubated overnight and resazurine (Sigma Aldrich, St Louis, MO, USA) staining $(0,1 \mathrm{~g} / \mathrm{L})$ was used as described to provide the minimal inhibitory concentration $(\mathrm{MIC})^{7}$ : the solution was prepared in sterile water, and then, briefly, $20 \mu \mathrm{L}$ of it was added to each well. Plates were kept in the dark for 10 $\min$ in home temperature. The lowest concentration in which resazurine (blue) was not converted to resofurin (pink) by microbial metabolism was considered as the MIC.

Qualitative assays consisted in agar diffusion tests. The pathogenic strains were seeded in BHI (Difco) agar plates using the spread-plate method, and two approaches were explored. On the first one, we spotted $20 \mu \mathrm{L}$ of each probiotic strain (of overnight grown cultures) prepared as a 0.5 McFarland suspension on each agar plate prepared with the bacterial pathogen seeded in it. On the second approach, we seeded the pathogens using the spread-plate method, made $6 \mathrm{~mm}$ holes in the agar and dispensed $50 \mu \mathrm{L}$ of the CFS in each. Inhibition zones were measured in plates prepared with the two approaches after overnight incubation at $37^{\circ} \mathrm{C}$.

All assays were performed in duplicate. ANOVA followed by Tukey post-hoc test was performed using Bioestat 5.0 for Windows for $\mathrm{E}_{24}$ experiments.

\section{RESULTS AND DISCUSSION}

Only the 72 hrs CFS of both strains was able to emulsify the hydrophobic substrates (Fig. 1). The statistical analysis indicated no significant difference among the CFSs, regardless of the tested substrate. However, we noted a tendency of better results with $L$. rhamnosus CFS.

None of the tested CFS of B. clausii presented antimicrobial activity in any of the tested methods. However, the 72 hrs CFS of $L$. rhamnosus presented antimicrobial activity in both methods. Inhibition zones were detected with the spotted strains $(10 \mathrm{~mm})$ and with the CFS $(18 \mathrm{~mm})$, and, in the semi-quantitative method, the CFS was active up to the $1 / 32$ dilution (data not shown). Superficial tension of water was reduced from $73 \mathrm{mN} / \mathrm{m}$ to 59.15 $\mathrm{mN} / \mathrm{m}$ by the CFS of B. clausii, and to 51.22 $\mathrm{mN} / \mathrm{m}$ by the CFS of L. rhamnosus.

Surface active compounds (SACs) are molecules that may alter the interaction of gasliquid, solid-liquid, and immiscible liquids surfaces such as water and oil, creating stable emulsions as shown in this study. SACs are divided in two main groups: low molecular weight, the biosurfactants, and high molecular weight, the bioemulsifiers. Biosurfactants are amphiphilic heteropolymers, generally composed of carbohydrates, proteins and fatty acids, which can decrease the surface and interfacial tensions of liquids, and form emulsions $^{1 \& 4}$. Bioemulsifiers are somehow similar to biosurfactants regarding the biochemical composition, and also can emulsify immiscible liquids and solubilize substrates; however, they are not efficient as biosurfactants to decrease surface tension of liquids ${ }^{7}$.

Although we have not characterized the SACs at the CFSs, it is possible that these are biosurfactants. Previous studies with $L$. rhamnosus and B. clausii indicated that these species are producers of biosurfactants: $L$. rhamnosus is known for producing rhamnolipid, and B. clausii for surfactin ${ }^{2 \& 4}$. Due to variations on cultivation conditions, SACs of different composition and biological properties can be produced ${ }^{6}$. Further studies are being conducted to characterize the SACs found in the tested CFS. 
Here, the CFS of $B$. clausii was ineffective against bacterial clinical isolates. A recent study described the first evidence of antimicrobial activity of the CFS of $B$. clausii from the same commercial formulation ${ }^{8}$. However, the bacterial culture was exposed to tetracycline for $120 \mathrm{hrs}$ before colleting the CFS, and metabolites of the drug were detected by HPLC $^{8}$. Thus, it is not clear if the pure $B$. clausii CFS cultivated in the conditions of the mentioned study could present antimicrobial activity. Our evidences indicate that, in our experimental conditions, B. clausii pure CFS lacks antimicrobial activity.

L. rhamnosus CFS was effective in semiquantitative and qualitative methods, as expected. Beyond biosurfactants, L. rhamnosus are known for their production of antimicrobial peptides known as bacteriocins ${ }^{4}$, which could be present at the culture media. A previous study of our group described the antibiofilm potential of the CFS of the strain used in the present study ${ }^{9}$. Also, a recent study investigated the antimicrobial potential of the CSF of lactobacilli from yogurt samples prepared with cow milk against extendedspectrum $\beta$-lactamase producing clinical isolates of Klebsiella pneumoniae and $P$. aeruginos $a^{10}$. The CFS was active against both species in agar diffusion test, inhibited biofilm formation and removed $24 \mathrm{hrs}$ old biofilms.

Curiously, there was no statistical difference on the effectiveness of $L$. rhamnosus and B. clausii CFS in the $\mathrm{E}_{24}$ assay. Although bioremediation studies are usually based in strains isolated from damaged areas (water or soil), such approach can be expensive, timeconsuming and cumbersome. Thus, exploring known probiotic strains can be a faster way to investigate potential alternatives for bioremediation. More studies will be conducted with the purified SAC from these species.

\section{Conclusion}

In this preliminary study, the CFS of commercial probiotic strains was effective in emulsifying hydrophobic substrates, but only $L$. rhamnosus presented antimicrobial activity. Further studies to characterize the SACs present in the CFS of these strains will be conducted in order to explore bioremediation options using B. clausii, in spite of the lack of antimicrobial activity in our in-vitro models.

\section{Acknowledgements}

We are thankful to Luiz Felipe Machado, Lorena Alcântara and Isabela Ceravolo for their contributions during the experiments. This research was supported by Pitagoras Faculty, Ipatinga, MG.

\section{REFERENCES}

1- S. Fijan, A. Frauwallner, T. Langerholc, et al., "Efficacy of using probiotics with antagonistic activity against pathogens of wound infections: An integrative review of literature", BioMed. Research Int., 2019, Article ID 7585486 (2019).

2- A. Upadrasta, S. Pitta, R. S. Madempudi, "Draft genome sequence of Bacillus clausii UBBC07, a spore-forming probiotic strain", Genome Announc., 4 (2), e00235-16 (2016).

3- L. D. C. Oliveira, A. M. M. Silveira, A. D. S. Monteiro et al., "In-silico prediction, in-vitro antibacterial spectrum, and physicochemical properties of a putative bacteriocin produced by Lactobacillus rhamnosus strain L156.4.", Front. Microbiol., 8, Article No. 876 (2017).

4- M. Zommiti, M. G. J. Feuilloley and N. Connil, "Update of probiotics in human world: A nonstop source of benefactions till the end of time", Microorganisms, 8 (12), 1907 (2020).

5- A. S. Monteiro, V. S. Domingues, M. V. D. Souza. et al., "Bioconversion of biodiesel refinery waste in the bioemulsifier by Trichosporon mycotoxinivorans CLA2", Biotechnol. Biofuels, 5, Article No. 29 (2012).

6- S. Viramontes-Ramos, M. C. PortilloRuiz, M. L. Ballinas-Casarrubias, et al., "Selection of biosurfactan/bioemulsifierproducing bacteria from hydrocarboncontaminated soil", Braz. J. Microbiol., 41 (3), 668-675, (2010).

7- M. V. Dias-Souza, S. Andrade, A. P. Aguiar and A. S. Monteiro. "Evaluation of antimicrobial and anti-biofilm activities of Anacardium occidentale stem bark extract", J. Nat. Prod., 26, 198-205 (2013). 
8- C. X. Liu, Q. M. Xu, S. C. Yu, et al., "Bio-removal of tetracycline antibiotics under the consortium with probiotics Bacillus clausii $\mathrm{T}$ and Bacillus amyloliquefaciens producing biosurfactants", Sci. Total Environ., 710, Article No. 136329 (2020).

9- R. F. Silva and M. V.Dias-Souza, "Controle de biofilmes em instrumental odontológico com probióticos", JAPHAC,
Special Edition: Annals of the I IJCPHS, 27-28 (2020).

10- M. A. El-Mokhtar, K. M. Hassanein, A. S. Ahmed, et al., "Antagonistic activities of cell-free supernatants of lactobacilli against extended-spectrum $\beta$-lactamase producing Klebsiella pneumoniae and Pseudomonas aeruginosa". Infect. Drug Resist., 13, 543-552 (2020). 


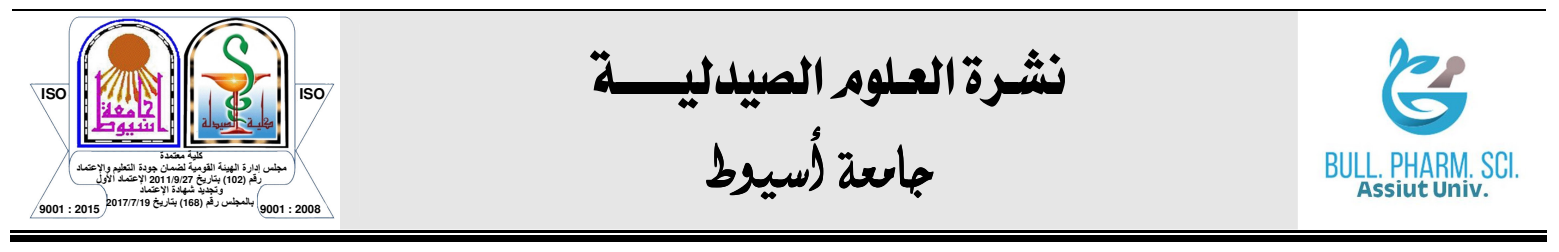

\section{التحقيق الأولي في إنتاج مركبات نشطة السطح مع خصائص مضادة للميكروبات}

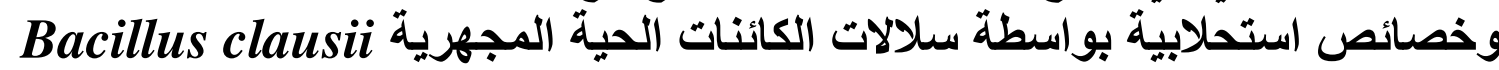
و Lactobacillus rhamnosus

تياجو سانتوس فونسيكا' - رينان مارتينز دوس سانتوس '، - ماركوس فينيسيوس دياس سوزا'، '، ' بلية بيتاغورس ، إيباتينجا ، البرازيل †المجموعة المتكاملة لأبحاث علم الأدوية والتفاعلات الدوائية (GPqFAR) ، البرازيل

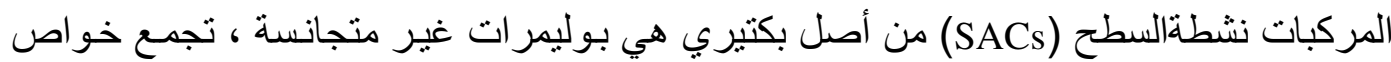

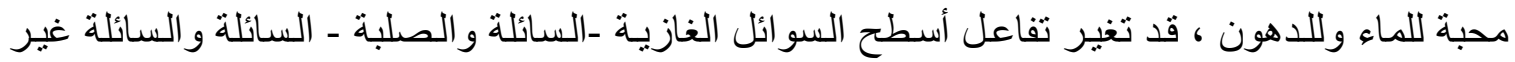

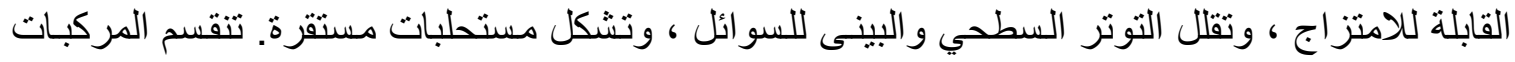

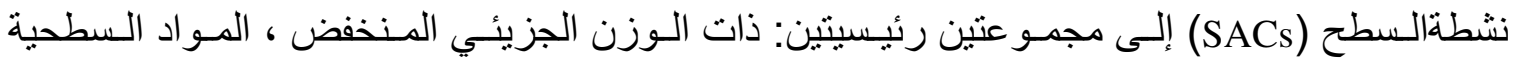

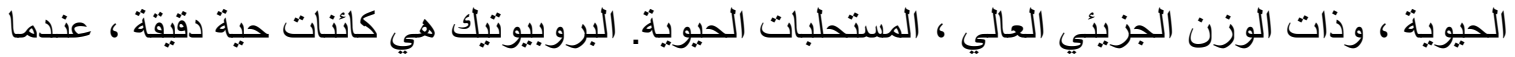

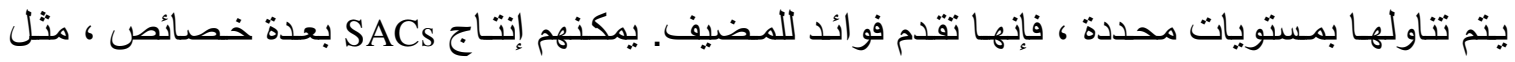

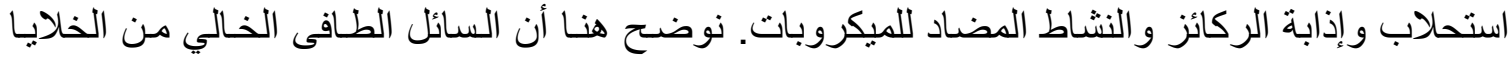

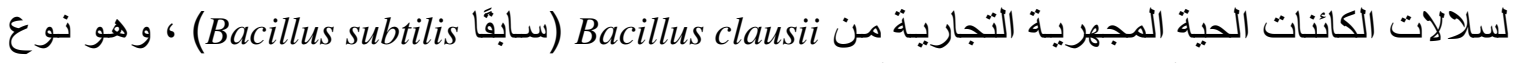

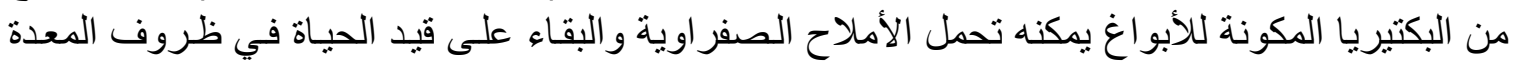

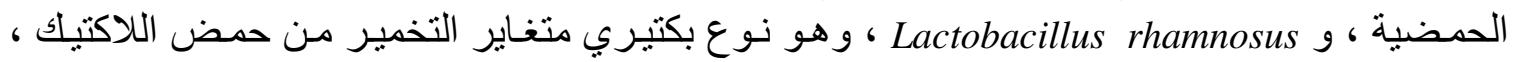

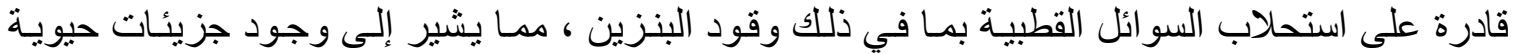

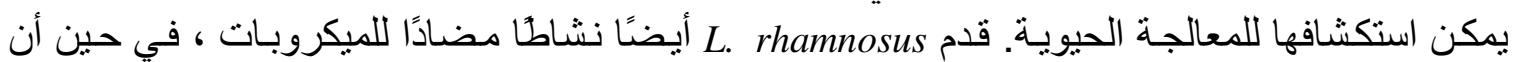
Bacillus clausii 